\title{
ACTAS DEL TALLER: CONOCIMIENTO Y VALORACIÓN DE LAS TURBERAS DE LA PATAGONIA: OPORTUNIDADES Y DESAFÍOS (24 - 25 DE NOVIEMBRE 2011, PUNTA ARENAS)
}

\author{
PROCEEDINGS OF WORKSHOP: KNOWLEDGE AND ASSESSMENT \\ OF THE PATAGONIAN'S PEATLANDS: OPORTUNITIES AND \\ CHALLENGES (24 \& 25 NOVEMBER, 2011, PUNTA ARENAS)
}

Ariel Valdés-Barrera ${ }^{1,2}$, Fiorella Repetto ${ }^{1}$, Alejandra Figueroa ${ }^{3} \&$ Bárbara Saavedra $^{1}$

\begin{abstract}
Peatlands are wetlands of global importance due to their capacity of storing huge amounts of carbon and water, as well as for housing a high percentage of exclusive flora and fauna. Only $4 \%$ of global peatlands are in South America, mainly in Chilean and Argentinean Patagonia. Worldwide, peatlands are threatened directly and indirectly by human activity. Sphagnum- a moss- key component of Patagonian peatlands, along with the peat that results from its partial decomposition- are highly demanded in a whole range of industries for their capacity to retain up to 20 times of its dry weight in water, as well as for other uses such as filtering. In Chile, peat is considered a mineral and its exploitation is under the Mining law. Furthermore, Patagonian peatlands are exposed to other threats such as draining and habitat modification, invasion of introduced species, climate change and lack of appreciation. Taking into consideration this critical situation, Wildlife Conservation Society, and the Chilean Ministry of Environment convened the Workshop "Knowledge and appreciation of Patagonian peatlands: Opportunities and Challenges" (November $24^{\text {th }}$ and $25^{\text {th }} 2011$ ) in Punta Arenas, Chile. The workshop offered the opportunity for updating on current knowledge of Patagonian peatlands, and on discussing guidelines regarding its conservation, research and regulation. The discussion highlighted the need for basic and applied research on peatlands, an inventory of Patagonian wetlands, and the need for developing outreach and education to increase their appreciation. A Binational Conservation Strategy and improved regulation allowing to deal with the complexity of these ecosystems as a whole are also deemed necessary.

Key words: Patagonia, peatlands, peatbogs, wetlands, Sphagnum, peat.

1 Wildlife Conservation Society - Programa Chileno - Avda. Gral. Bustamante 126 Of. 42, Providencia, Santiago.

2 Laboratorio de Ecología de Ecosistemas, Facultad de Ciencias Agronómicas, Universidad de Chile. Santa Rosa 11315, La Pintana, Santiago.

3 Encargada Humedales, División Recursos Naturales, Ministerio del Medio Ambiente, Santiago, Chile.

1 Catastro y levantamiento geológico de Turba en Chiloé; región de Los Lagos, SERNAGEOMIN-GORE Los Lagos, Agosto 2008.
\end{abstract}




\section{RESUMEN}

Las turberas son humedales de importancia global, cuya biodiversidad singular le otorga una gran capacidad de almacenar carbono y agua, y sostener especies de flora y fauna únicas las que tiene gran valor ecológico y paisajístico. En América del Sur se encuentra sólo un 4\% de ellas, principalmente en Chile y Argentina, las que están bajo amenazas variadas. El musgo Sphagnum, componente principal de las turberas Patagónicas, es requerido en la industria mundial por su alta capacidad de retención de agua. La turba, materia orgánica en descomposición que se deposita y origina estos ecosistemas, se considera una materia fósil, y por lo mismo su explotación es permitida bajo la Ley Minera. Otras amenazas globales que afectan a las turberas son; la invasión de especies exóticas, cambio climático, y especialmente la falta de conocimiento y valoración de los bienes y servicios ecosistémicos que ellas proveen. Con el objetivo de promocionar el conocimiento y valor de estos ecosistemas, los días 24 y 25 de noviembre de 2011, se realizó en Punta Arenas el Taller "Conocimiento y valoración de las turberas de la Patagonia: Oportunidades y desafíos", iniciativa conjunta de Wildlife Conservation Society \& el Ministerio del Medio Ambiente de Chile. El Taller tuvo por objetivo inicial actualizar sobre el conocimiento de las turberas de Chile y Argentina, para luego en una segunda etapa más participativa, discutir lineamientos para su conservación, investigación y normativa de estos humedales a nivel Binacional. Dentro de los principales resultados del Taller, se destaca la necesidad de realizar investigación básica y aplicada de las turberas, un catastro integral que permita la clasificación de estos humedales, que contemple la Patagonia chilena, ya que actualmente sólo podemos dar cuenta de una pequeña porción del territorio, además de educación y divulgación para incrementar su valoración. También se destacó la necesidad de elaborar una Estrategia de Conservación Binacional y una actualización de la normativa vigente que permita abordar la complejidad de estos ecosistemas, así como el Plan de acción de la Estrategia Nacional de humedales de Chile, CONAMA 2006.

Palabras clave: Patagonia, humedal, turberas, Sphagnum, turba.

\section{ANTECEDENTES}

Las turberas son un tipo de humedal que se caracteriza por la producción progresiva de turba, material que resulta de la lenta descomposición y compactación de la materia orgánica de la vegetación dominante. Las turberas son reconocidas internacionalmente como uno de los mayores sumideros de carbono del planeta. Su superficie abarca entre 3-6\% de la superficie de la Tierra (Clymo, 1984, Gorham, 1991); sin embargo, almacenan un tercio del $\mathrm{C}$ de los suelos terrestres, lo que equivale a un 75\% del C contenido en la atmósfera (Gorham, 1991, Shurpali et al. 2008, Vitt et al. 2000, Asada \& Warner, 2005). Además, las turberas almacenan el 10\% del agua dulce disponible, de hecho, turberas saturadas contienen 90-98\% de su masa en agua (Päivänen, 1982). Esta característica le confiere a las turberas la capacidad de regular el flujo hídrico a escalas de cuenca, interceptando el escurrimiento y almacenando las aguas pluviales (Holden, 2005).
Este tipo de ecosistema alberga una flora y fauna propia y característica, capaz de vivir en condiciones que son adversas para otras especies (van Breemen, 1995) como el constante anegamiento, acidez, anoxia y escasa disponibilidad de nutrientes.

La distribución mundial de las turberas es altamente heterogénea concentrándose la mayor parte en el hemisferio norte, mientras que solo un 4\% se ubica en América del Sur, principalmente en la Patagonia de Chile y Argentina. Las turberas en esta zona pertenecen al complejo Tundra Magallánica y se encuentran dominadas por musgos del género Sphagnum característicos de hábitats húmedos, suelos de alta acidez y climas templadosfríos (Pisano, 1977, 1983). En esta región, existen amenazas directas e indirectas, que afectan la persistencia de las turberas en el largo plazo, como la explotación minera para extracción de turba y la cosecha de musgo, el cambio climático, así como también la falta de conocimiento y valoración de 
estos ecosistemas australes como componentes relevantes de la biodiversidad mundial.

En este contexto, los días 24 y 25 de Noviembre de 2011, se realizó en la ciudad de Punta Arenas (Chile) el Taller "Conocimiento y valoración de las turberas de la Patagonia: Oportunidades y desafíos", iniciativa gestada por la Wildlife Conservation Society (WCS) en conjunto con el Ministerio del Medio Ambiente (MMA) de Chile. El objetivo de este taller fue discutir y diseñar estrategias conjuntas que permitan avanzar en la implementación efectiva de programas de protección y conservación de las turberas patagónicas a una escala binacional (Argentina-Chile). Entre los participantes se encontraron miembros de las Secretarias Regionales del Ministerio de Medio Ambiente (SEREMI) de las regiones de Los Lagos y Magallanes, Corporación Nacional Forestal (CONAF), Servicio Agrícola y Ganadero (SAG), Instituto de Investigaciones Agropecuarias (INIA), Oficina de Estudios y Políticas Agrarias (ODEPA), Ministerio de Agricultura; Corporación Nacional Forestal (CONAF), Subsecretaría de Medio Ambiente y Recursos Naturales de Tierra del Fuego, Argentina; representantes de organizaciones no gubernamentales como Wetlands International, Corporación Instituto de Ecología y Biodiversidad (IEB) y la Fundación Centro de Estudios del Cuaternario; representantes de instituciones privadas como Patagonia Peat, Centro de Ecología Aplicada (CEA) y Patagonia Wildlife, científicos de distintas universidades procedentes de Chile, Argentina, Suecia, Nueva Zelandia y España; y propietarios de predios en la región de Magallanes.

\section{ENFOQUE METODOLÓGICO}

El taller de expertos en Chile tuvo como objetivos específicos: 1. dar a conocer la existencia, extensión, valor, y problemas asociados a la conservación de las turberas patagónicas; 2 . identificar su aporte a escala local y global valorar su conservación, viabilidad para su uso sustentable.

La convocatoria se extendió a distintas instituciones públicas y privadas relacionadas directa o indirectamente con el uso, explotación y conservación de las turberas en Chile y Argentina, junto a los profesionales y especialistas en el tema. El taller contó con la participación de 66 asistentes (Anexo
1) y se dividió en dos partes: una primera expositiva (Anexo 2), cuya función fue actualizar a los asistentes sobre el estado del conocimiento y valoración. Los resúmenes de cada presentación se adjuntan en el Anexo 3. La segunda parte fue participativa, pues se conformaron tres grupos de discusión en torno a las temáticas generales de Investigación y Monitoreo, Conservación y Uso Sustentable, y Política y Normativa. Cada grupo de discusión identificó aspectos críticos, propios y comunes a Chile y Argentina, y estructuró líneas de trabajo, identificando potenciales pilares para futuros acuerdos binacionales, o para la solicitud de apoyo a instituciones internacionales. Los resultados y conclusiones de cada grupo de discusión se presentaron al cierre del taller y se presentan resumidamente a continuación.

\section{GRUPO DE DISCUSIÓN: INVESTIGACION Y MONITOREO}

El Grupo indicó que antes de iniciar estudios para la clasificación de las turberas se requiere unificar criterios para adoptar definiciones científicas únicas, que permitan su reconocimiento y delimitación espacial. Estos criterios deben considerar no sólo la vegetación dominante, sino también causa de origen, geomorfología, así como el aporte hídrico de la turbera. Además, se debe promover el desarrollo de talleres locales que apunten al perfeccionamiento de profesionales, con el objetivo de avanzar en el inventario de las turberas, así como también en el desarrollo de una propuesta de ordenamiento territorial en Chile, utilizando como ejemplo el modelo de Argentina para la localidad de Tierra del Fuego.

Respecto a la investigación en turberas, se sugirió fomentar el estudio a largo plazo de los procesos ecosistémicos, principalmente la regulación del ciclo del agua y el balance de carbono, los cuales se ven seriamente amenazados producto de la explotación minera y el cambio climático. Se reconoció la urgencia de priorizar estudios sobre restauración de turberas intervenidas, debido a la falta de experiencias positivas de restauración ecológica para el cono sur de América. La experiencia del hemisferio norte nos indica que la explotación de estos ecosistemas genera alteraciones significativas en las condiciones físicas e hidrológicas del ambiente (Heathwaite, 1994, Price, 1996), debido que la 
remoción de la cobertura vegetal fotosintéticamente activa, el drenaje y la extracción absoluta del suelo orgánico, son difíciles de recuperar por sí solas, y estas influyen directamente sobre la biodiversidad presente y regulan los procesos ecosistémicos que permiten la mantención y sostenibilidad de las turberas en el tiempo.

\section{GRUPO DE DISCUSIÓN: CONSERVACION Y USO SUSTENTABLE}

Se reconoció la necesidad de diseñar una estrategia binacional, entre Chile y Argentina, que considere un plan de investigación, ordenamiento territorial y discusión entre los distintos actores involucrados. Se propuso identificar distintos actores y los principales conflictos asociados al uso de las turberas. Para facilitar lo anterior, se sugirió desarrollar una plataforma de comunicación, liderada idealmente por el Ministerio del Medio Ambiente, que permita recopilar y exponer información, además de contactar y coordinar a los distintos actores en la región. Es primordial durante este proceso, realizar un análisis bio-económico de las ganancias y pérdidas asociadas a la explotación minera de las turberas, lo cual requiere de una adecuada valoración de los bienes y servicios ecosistémicos proporcionados por estos ecosistemas. Para lo anterior, se recomienda utilizar los criterios y la clasificación propuesta por la Evaluación Ecosistémica del Milenio (Millenium Ecosystem Assessment).

También se propone evaluar otras alternativas de uso no extractivo, como el turismo, pago por servicios ecosistémicos, bonos de carbono y agua, que puedan servir para generar incentivos económicos para la conservación de las turberas.

A modo de estrategia para la conservación de este tipo de humedal, se indicó la necesidad de identificar áreas relevantes para la conservación de los ecosistemas de humedales y biodiversidad asociada, así como aquellas frágiles o degradadas, asimismo es necesario identificar el grado de vulnerabilidad y los procesos y funciones de importancia local.

Para alcanzar las metas consensuadas en conservación es relevante, a nivel nacional, adecuar la legislación y normativa ambiental vigente.

\section{GRUPO DE DISCUSIÓN: POLITICA Y NORMATIVA}

La adecuación de legislación y normativa ambiental en Chile es esencial para cumplir con las metas de conservación de las turberas en la región. Si bien las turberas son incluidas en la Ley № 19.300 de Bases Ambientales, su definición es sencilla y no explicita los componentes, estructura y procesos de este tipo de ecosistema, por lo que se indicó que se requiere una modificación que permita avanzar en esto. Además, se propuso diferencia en el reglamento del Sistema de Evaluación de Impacto Ambiental (SEIA), aquellas turberas naturales de los "pomponales" o turberas de origen antrópico cuya formación es más reciente (Díaz et al. 2007, Valenzuela-Rojas \& Schlatter 2004) y cuya distribución se restringe principalmente al archipiélago de Chiloé.

Se indicó que es razonable pensar que las turberas deberían dejar de ser consideradas sólo como fuente de un recurso minero, como señala la Ley Orgánica de Minería, y deberían ser reconocidas como un ecosistema, en el cual se integran, componentes bióticos y abióticos que interactúan entre sí. Dada la complejidad que representan las turberas y sus diferencias con otros ecosistemas, se discutió que probablemente sea necesario dictar una ley específica para este tipo de humedal.

Se indicó que es importante recordar que Chile es un país signatario de la Convención Ramsar, por lo que se deben seguir los lineamientos internacionales para la protección y conservación de humedales, entre los cuales se encuentran las turberas.

Por otra parte, se indicó que para reducir el impacto de los proyectos en ejecución que contemplan la extracción de turba y musgo, se debería desarrollar e implementar un manual de buenas prácticas, que indique algunas acciones concretas para evitar la pérdida absoluta del suelo orgánico, vegetación, procesos y funciones características de estos ecosistemas con la finalidad permitir la restauración luego de ser explotado. Para constatar el seguimiento de este manual se propuso crear un sistema de certificación ambiental, con un sello reconocible para los consumidores de los productos derivados de la turba y musgo.

Finalmente, como una herramienta para relevar el valor de las turberas, se sugirió incorporar temáticas alusivas a este tipo de ecosistemas en la 
malla curricular de los estudiantes de educación primaria y secundaria.

\section{CONCLUSIONES}

Durante el Taller y en particular durante el trabajo de los grupos de discusión, fue posible identificar las principales brechas para alcanzar la conservación de turberas, y fue una opinión general la necesidad de generar capacidades para el estudio, catastro y valoración de las turberas Patagónicas. Dada la naturaleza compleja de estos ecosistemas, este trabajo debería ser multidisciplinario. Se mencionó además que tal como ocurre con otros ecosistemas de importancia estratégica como los bosques o ecosistemas marinos, debería existir financiamiento estatal para avanzar en investigaciones, innovación tecnológica, educación y divulgación, y capacitación profesional.

Los participantes a este Taller indicaron la necesidad de coordinar las actividades entre los distintos Ministerios competentes además de sus Servicios asociados, incluyendo por ejemplo al Ministerio del Medio Ambiente, Ministerio de Agricultura, Ministerio de Minería y Ministerio de Obras Públicas. Más aún, se indicó la necesidad de re-distribuir atribuciones sobre este tipo de ecosistema; ya que, si bien existe una legislación vigente alusiva a las turberas en Chile, esta se encuentra atomizada y no aborda el problema en su complejidad.

Con respecto a los planes de ordenamiento territorial, investigación, manejo y conservación de las turberas, todos los grupos de discusión proponen desarrollar una estrategia binacional de conservación entre Chile y Argentina tomando como guía las experiencias positivas de países como Nueva Zelandia, Suecia y Canadá, entre otros. En este sentido, el taller "Conocimiento y valoración de las turberas de la Patagonia" constituye un hito no sólo por que permitió el acercamiento y discusión entre los distintos actores e instituciones locales, sino también porque aportó a la generación de vínculos de cooperación en estas materias entre Chile y Argentina, además de especialistas provenientes de distintas latitudes.

\section{AGRADECIMIENTOS}

Agradecemos el apoyo financiero del IEB y CEA, también a la Universidad de Magallanes y al
Instituto de la Patagonia por el apoyo entregado, en especial por la gestión y facilitación de las dependencias del Centro Austral Antártico para llevar a cabo el Taller. En particular al Dr. Carlos Ríos, por la activa invitación a publicar las Actas en los Anales del Instituto de la Patagonia. También agradecemos a todas las personas e instituciones participantes del Taller, quienes hicieron posible avanzar en cuanto a los lineamientos necesarios para la conservación de las turberas en Chile y Argentina.

En particular agradecemos a Juan Marcos Henríquez, Ernesto Teneb \& Claudia Silva por apoyarnos en la materialización de estas actas.

\section{LITERATURA CITADA}

Asada, T. \& B.G. Warner 2005. Surface peat mass and carbon balance in a hypermaritime peatland. Soil Science Society of America Journal, 69:549-562.

Clymo, R. S. 1984. Limits to peat bog growth. Philosophical Transactions of the Royal Society of London, B, 303: 605-654.

Díaz, M. F, S. Bigelow \& J. J. Armesto 2007. Alteration of the hydrologic cycle due to forest clearing and its consequences for rainforest succession. Forest Ecology and Management, 244: 32-40.

Gorham, E. 1991. Northern Peatlands: Role in the Carbon Cycle and Probable Responses to Climatic Warming. Ecological Applications, 1: 182-195.

Heathwaite A. L. 1994. Hydrological management of a cutover peatland. Hydrological Processes, 8: 245-262.

Holden J. 2005. Peatland hydrology and carbon cycling: why small-scale process matters. Philosophical Transactions of the Royal Society A 363: 2891-2913.

Päivänen J. 1982. Main Physical Properties of Peat Soils. En Laine J (ed) Peatlands and their utilization in Finland (Helsinki, Finland: Finnish Peatland Soc. 1982): 33-36.

Pisano, E. 1977. Fitogeografía de Fuego-Patagonia Chilena. I. Comunidades vegetales entre las latitudes $52^{\circ}$ y $56^{\circ} \mathrm{S}$. Anales del Instituto de la Patagonia, 8: 121-250.

Pisano, E. 1983. The magellanic complex tundra. En Gore AJP (ed.) Mires, swamps, bog, fen 
and moor, $B$ Regional studies. (Ecosistems of the world 4B). Amsterdam, pp 295-329.

Price J. S. 1996. Hydrology and microclimate of a partly restored cutover bog, Quebec. Hydrological Processes, 10: 1263-1272.

Shurpali N. J., N. P. Hyvonen, J. T. Huttunen, C. Biasi, H. Nykanen, N. Pekkarinen \& P. J. Martikainen 2008. Bare soil and reed canary grass ecosystem respiration in peat extraction sites in Eastern Finland. Tellus B, 60: 200-209.

Valenzuela-Rojas J. \& R. Schlatter 2004. Las turberas de la Isla Chiloé (X Región, Chile): aspectos sobre usos y estado de conservación. En:
Blanco DE \& VM Balze (eds). Los Turbales de la Patagonia Bases para su inventario y la conservación de su biodiversidad, Publicación No. 19: 87-92. Wetlands International - América del Sur, Buenos Aires, Argentina. Van Breemen N. 1995. How Sphagnum bogs down other plants. Trends in Ecology and Evolution, 10: 270-275.

Vitt D. H., L. A. Halsey, I. E. Bauer \& C.Campbell 2000. Spatial and temporal trends in carbon storage of peatlands of continental western Canada through the Holocene. Canadian Journal of Earth Sciences, 37:683-693. 


\section{ANEXO 1}

\section{Listado de asistentes al Taller}

№ NOMBRE

1 Teresa Agüero

2 Marta Andelman

3 Juan Carlos Aravena

4 Nelson Bahamonde

5 Karina Bastidas

6 Ricardo Bennewitz

7 Sebastián Bertrand

8 Sol Bustamante

9 José Luis Cabello

10 Michelle Chaumenau

11 Manuel Contreras

12 Sergio Cornejo

13 Daniel Correa

14 Francois De Vleeschouwer

15 Guillermo Délano

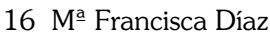

17 Erwin Domínguez

18 Daniela Droguett

19 Alejandra Figueroa

20 Hans Gardjell

21 Lennat Henrikson

22 Juan Marcos Henríquez

23 Daniel Iturraspe

24 Rodolfo Iturraspe

25 Cristóbal Ivanovich

26 Juan Ivanovich

27 Carolina León

28 Constanza León

29 Rita Lofiego

30 Diego López

31 Yanet Medina

32 Nelson Moncada

33 Claudio Moraga

34 Ricardo Muza

35 Fernando Novoa

36 Mariela Nuñez

37 Nelly Nuñez

38 Christel Oberpaur

39 José Ojeda

INSTITUCIÓN

Oficina de Estudios y Políticas Agrarias (ODEPA)

Wetlands International

PAÍS

GRUPO DE DISCUSIÓN

Centro de Estudios del Cuaternario Fuego-Patagonia Chile

y Antartica (CEQUA)

Instituto de Investigaciones Agropecuarias (INIA) Chile

Servicio de Evaluación Ambiental-Ministerio del Chile

Medio Ambiente (SEA-MMA), Región de Magallanes

Chile Política y Normativa

Argentina Política y Normativa

SEREMI (Secretaria Regional Ministerial) Agricultura Chile Región Magallanes y Antártica Chilena

University of Ghent,

SEREMI, MMA, Región de Los Lagos

Bélgica Investigación y Monitoreo

Patagonia Wildlife

Chile Conservación y Uso Sustentable

Chile Política y Normativa

Universidad de Magallanes (UMAG)

Chile

Centro de Ecología Aplicada (CEA) Chile

SEREMI MMA Región de Magallanes y Antártica Chile

Investigación y Monitoreo

Chilena

Corporación Nacional Forestal (CONAF)

University of Aberdeen

Universidad Santo Tomás (UST)

Universidad Andrés Bello (UNAB)

Instituto de Investigaciones Agropecuarias (INIA)

Wildlife Conservation Society (WCS)

Ministerio del Medio Ambiente (MMA)

Swedish University of Agricultural Sciences

Swedish University of Agricultural Sciences

\section{Chile}

Francia

Política y Normativa

Chile

Chile

Chile

Chile

Chile

Suecia

Suecia

Instituto de la Patagonia, Universidad de Magallanes Chile

Política y Normativa

Conservación y Uso Sustentable

Conservación y Uso Sustentable

Conservación y Uso Sustentable

Conservación y Uso Sustentable

Política y Normativa

Secretaría de Desarrollo Sustentable y Ambiente de Argentina Investigación y Monitoreo Tierra del Fuego

Secretaría de Desarrollo Sustentable y Ambiente de Argentina Investigación y Monitoreo Tierra del Fuego

Centro de Estudios del Cuaternario Fuego-Patagonia Chile y Antartica (CEQUA)

Corporación Nacional Forestal (CONAF)

Universidad de Complutense de Madrid

Oficina de Estudios y Políticas Agrarias (ODEPA)

Secretaría de Desarrollo Sustentable y Ambiente de Tierra del Fuego

Dirección de Vialidad, Región Magallanes

Parque Etnobotánico OMORA

Corporación Nacional Forestal (CONAF)

Wildlife Conservation Society (WCS)

Wildlife Conservation Society (WCS)

Centro de Ecología Aplicada (CEA)

Instituto de Ecología y Biodiversidad (IEB)

Ministerio del Medio Ambiente (MMA)

Universidad Santo Tomás (UST)
Chile

España Conservación y Uso Sustentable

Chile Política y Normativa

Argentina Política y Normativa

Chile

Chile

Chile

Chile

Chile

Chile

Chile

Chile

Chile

Chile
Conservación y Uso Sustentable

Investigación y Monitoreo

Investigación y Monitoreo

Investigación y Monitoreo

Política y Normativa 
40 Felipe Osorio

41 Verónica Pancotto

42 Patricio Pérez

43 Soledad Quiroz

44 Fiorella Repetto

45 José Luis Riffo

46 Tomás Rioseco

47 Hugh Robertson

48 José Ruiz

49 Bárbara Saavedra

50 Claudia Saavedra

51 Saskia Sandring

52 Alejandra Silva

53 Ángel Suárez

54 Johan Svensson

55 Ernesto Teneb

56 Eduardo Toloza

57 Patricio Toro

58 Toradj Uraoka

59 Adriana Urciolo

60 Javiera Vargas

61 Alberto Vera

62 Karina Vergara

63 Joaquín Vicuña

64 Rodrigo Villa

65 Eduardo Villouta

66 Dave West
Centro Austral de Investigaciones Científicas (CADIC)

Cuerpo Militar del Trabajo (CMT)

Universidad Santo Tomás (UST)

Wildlife Conservation Society (WCS)

Centro de Ecología Aplicada (CEA)

Department of Conservation

Patagonia Peat

Wildlife Conservation Society (WCS)

SEREMI MMA Magallanes

Swedish University of Agricultural Sciences

Corporación Nacional Forestal (CONAF)

Instituto de Investigaciones Agropecuarias (INIA)

Swedish University of Agricultural Sciences

Instituto de la Patagonia - Grupo de Estudios

Ambientales (GEA)

Dirección de Vialidad, Región de Magallanes

Centro de Ecología Aplicada (CEA)

Secretaría de Desarrollo Sustentable y Ambiente de Tierra del Fuego

$\begin{array}{lr} & \text { Chile } \\ & \text { Chile } \\ \text { Corporación Nacional Forestal (CONAF) } & \text { Chile } \\ \text { Oficina de Estudios y Políticas Agrarias (ODEPA) } & \text { Chile } \\ \text { Centro de Estudios del Cuaternario Fuego-Patagonia Chile } \\ \text { y Antártica (CEQUA) }\end{array}$

Department of Conservation
Nueva

Zelandia

Nueva Investigación y Monitoreo

Argentina Investigación y Monitoreo

$\begin{array}{ll}\text { Chile } & \\ \text { Chile } & \text { Política y Normativa } \\ \text { Chile } & \text { Política y Normativa } \\ \text { Chile } & \text { Conservación y Uso Sustentable } \\ \text { Chile } & \text { Conservación y Uso Sustentable } \\ \text { Nueva } & \text { Conservación y Uso Sustentable } \\ \text { Zelandia } & \\ \text { Chile } & \text { Conservación y Uso Sustentable } \\ \text { Chile } & \\ \text { Chile } & \\ \text { Suecia } & \text { Investigación y Monitoreo } \\ \text { Chile } & \text { Conservación y Uso Sustentable } \\ \text { Chile } & \text { Investigación y Monitoreo } \\ \text { Suecia } & \text { Investigación y Monitoreo } \\ \text { Chile } & \text { Investigación y Monitoreo }\end{array}$

Argentina Política y Normativa

Chile Política y Normativa

Chile

Política y Normativa

Investigación y Monitoreo

Política y Normativa

Zelandia 


\section{ANEXO 2}

\section{Listado de exposiciones presentadas durante el Taller}

1 Ordenamiento del uso de las turberas en Tierra del Fuego

2 Las turberas como reservorios de información paleoambiental

3 Turberas Patagónicas: su valor global y desafíos para la conservación

4 Cambio global. Efectos y aportes de las turberas de Tierra del Fuego

5 Turberas en Magallanes (Chile), situación actual, conservación y explotación Erwin Domínguez \& Nelson Bahamonde para su uso y conservación

6 High nature conservation values in wetland and peatland habitats - developing Hans Gardfjell approaches for long term monitoring of habitats according to the EC Species and Habitats Directive

7 Parad-Patagon project and field mission

8 Ecología de pomponales de Chiloé

9 Plan de manejo sustentable y modelo de fiscalización para humedales con predominio de musgo pompón (Sphagnum magellanicum) en las provincias de Llanquihue y Chiloé

10 Caracterización de servicios ecosistémicos de turberas de la Isla Grande de Chiloé, una herramienta para la conservación

11 Generación de bases técnicas para el desarrollo de un Plan de manejo sustentable y Modelo de fiscalización de Musgo Sphagnum

12 Aspectos normativos claves relacionados con las turberas, un tema pendiente Joaquín Vicuña

13 Enfoque eco-hidráulico para el estudio y manejo de turberas: experiencias Manuel Cotreras en humedales andinos

14 National inventory of landscapes in Sweden (NILS): Wetlands and peatlands Saskia Sandring in Sweden

15 New Zealand Peatland Conservation experience and knowledge integration Hugh Robertson \& David West
Adriana Urciuolo \& Rodolfo Iturraspe

Rodrigo Villa-Martínez, Héctor Mansilla, Juan

Carlos Aravena \& Erwin Domínguez

Marta Andelman

François De Vleeschouwer

Ma Francisca Díaz

Mª Francisca Díaz \& Guillermo Delano

Carolina León Valdebenito, G. Oliván

Martínez \& E. Fuertes Lasala

Guillermo Délano 


\title{
ANEXO 3
}

Resúmenes de algunas de las presentaciones orales del Taller

\section{ORDENAMIENTO DEL USO DE LAS TURBERAS EN TIERRA DEL FUEGO}

\author{
Adriana Urciuolo \& Rodolfo Iturraspe"
}

Las turberas, que frecuentemente son consideradas simplemente un recurso económico vinculado a la minería, son además recursos hídricos, ya que conforman humedales con gran capacidad de regulación hidrológica. Son también ecosistemas que sustentan biodiversidad, por lo que constituyen un recurso biológico y tienen un valor económico que trasciende su importancia como recurso minero, basta para ello considerar su importancia como atractivo turístico paisajístico propio del extremo austral de Sudamérica, donde la actividad turística, basada en los escenarios naturales, tiene un peso importante en la economía. Son además reguladoras del clima global, y reservorios paleoclimáticos que guardan la historia ambiental del Holoceno, de gran utilidad científica. En síntesis, son múltiples sus valores, así como las funciones ambientales que ofrecen. En la Provincia de Tierra del Fuego Argentina, la extracción de turba como actividad económica productiva primaria comenzó en 1970 y se extendió progresivamente bajo la regulación de la Ley Nacional de Minería, sin que en principio el Estado Provincial asumiera un rol definido en la planificación del uso del recurso. La proliferación de pedimentos para uso extractivo, y el carácter precario e improvisado de emprendimientos de incierta capacidad productiva pero que implicaban la degradación de las turberas intervenidas, generaron la necesidad de implementar un plan que contemple el ordenamiento del uso de las mismas. Para ello se consideró no orientar tal planificación según el beneficio de un sector particular de la economía en detrimento de otros y del propio ambiente, sino responder al sentido común y a un enfoque racional, optimizando los beneficios que el Estado Provincial y la sociedad en su conjunto pudiese obtener de ellos, considerando además los valores ambientales. El plexo normativo ambiental, conformado por leyes nacionales y provinciales otorga al Estado Provincial la potestad de planificar el uso de las turberas y en función de ello se ha seguido un largo proceso participativo que culminó en una serie de medidas, entre las que resulta de relevancia la zonificación del uso de las turberas. Tal zonificación resultó del trabajo de la Comisión para el Ordenamiento de Turberas, integrada por diferentes áreas de la Secretaría de Desarrollo Sustentable y Ambiente de la Provincia con injerencia en este tema: Minería, Recursos Hídricos, Gestión Ambiental, Bosques, Áreas Protegidas, Planificación Territorial, etc.

La zonificación se basó en criterios consensuados en talleres participativos, agrupados en: a) planes y medidas persistentes, b) aspectos sociales y económicos, c) aspectos hidrológicos, y d) aspectos ecosistémicos. En base a estos criterios se delimitaron tres diferentes categorías de uso: una zona de sacrificio o con habilitación regulada del uso extractivo, zonas de protección y zonas protegidas, en reserva por 30 años, con potencialidad para el futuro uso extractivo. La norma correspondiente es la Resolución SD 401/2011 emitida por la Secretaría de Desarrollo Sustentable y Ambiente. 


\title{
LAS TURBERAS COMO RESERVORIOS DE INFORMACIÓN PALEOAMBIENTAL
}

\author{
R. Villa-Martínez", H. Mansilla*", J. C. Aravena* \& E. Domínguez"*
}

Presentamos los resultados de dos registros de polen provenientes las turberas Lago Parrillar

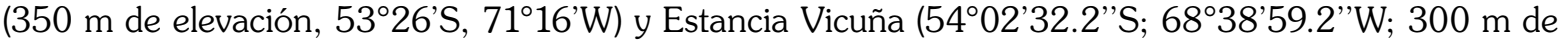
elevación), con el propósito de evaluarlas como reservorios de información paleoambiental y reconstruir la evolución del paisaje y clima en la Región de Magallanes. Estos sitios se localizan cerca de los actuales ecotonos entre el bosque siempreverde-deciduo y bosque-estepa. Estos límites naturales de la vegetación dependen del gradiente de precipitación, por lo que son ideales para reconstruir variaciones pasadas de los vientos del oeste. Los registros muestran la presencia de lagos en un paisaje dominado por herbáceas y escasos elementos del bosque magallánico, bajo condiciones climáticas frías y húmedas. El cambio sedimentológico desde limos lacustres a turba, junto con el incremento sostenido de Nothofagus sugiere descenso de niveles lacustres, formación de turberas, y expansión del bosque bajo condiciones climáticas más cálidas y secas que el lapso previo. La comparación de este cambio vegetacional con otros registros paleoecológicos disponibles en la región, sugiere que este cambio ocurrió durante la transición PleistocenoHoloceno ( 12.000-10.500 años AP). Luego de este cambio, Nothofagus alcanza valores máximos y se mantiene con pocas variaciones hasta la actualidad, marcando el establecimiento del bosque magallánico. Sin embargo, variaciones en los sedimentos durante la primera mitad del Holoceno, sugieren periodos de mayor humedad. Estos cambios no afectaron la composición ni abundancia de los indicadores polínicos. Las variaciones de la precipitación inferidas en este registro sugieren mayor influencia de vientos del oeste a fines del Pleistoceno, un debilitamiento durante los primeros milenios del Holoceno, seguido por un fortalecimiento desde entonces. Estos antecedentes implican que las turberas de la región constituyen importantes reservorios de información paleoambiental.

* Centro de Estudios del Cuaternario (Fundación CEQUA), Punta Arenas.

** Instituto de Investigaciones Agropecuarias (INIA-Kampenaike), Punta Arenas. 


\section{TURBERAS EN MAGALLANES (CHILE), SITUACIÓN ACTUAL, CONSERVACIÓN Y EXPLOTACIÓN PARA SU USO Y CONSERVACIÓN}

Domínguez, E." \& Bahamonde, N."

Las turberas son un tipo especial de humedales, que están integrados por una gran variedad de organismos. Además representan un recurso económico de valor desconocido localmente a excepción de la extracción de turba y de la fibra de Sphagnum conocida también como pompón. Diversos estudios han sugerido que estos ecosistemas, cumplen importantes funciones que benefician al medio ambiente. Considerando estos antecedentes, la SEREMI de Agricultura de la Región de Magallanes ha decidido abordar los problemas ligados al aprovechamiento de este importante recurso en la región. A partir de esta iniciativa se inicia la formulación de este programa, que cuenta con el apoyo del Gobierno Regional de Magallanes y Antártica Chilena y es financiado por el Fondo para el Desarrollo de Magallanes (FONDEMA). La unidad técnica es la SEREMI de Agricultura y la institución ejecutora es el INIA-Centro Regional de Investigación Kampenaike. Este programa pretende generar las bases científico-ambientales para la elaboración y puesta en marcha de una propuesta política y de legislación para el desarrollo sustentable de las turberas de la Región de Magallanes. La presentación del INIA se enfocó en mostrar los resultados preliminares de dos ensayos de restauración en una turbera Sphagnum en donde actualmente se cosecha turba en bloques, ubicada en San Juan, a $60 \mathrm{~km}$ al sur de Punta Arenas. Los ensayos se establecieron en dos situaciones de post-cosecha de turba: 1) zanjas con residuos vegetales (llamado escarpe) depositados superficialmente en el centro y 2) zanjas sin escarpe. En ambas situaciones se implantaron diásporas de $S$. magellanicum $( \pm 10 \mathrm{~cm}$ ) de sitios donantes cercanos, los que fueron cubiertos con una capa protectora vegetal y una malla. Los primero brotes emergentes demoraron entre 9 y 18 meses. Se encontró que la respuesta del Sphagnum implantado en el escarpe, se retarda más (18 meses) debido a la profundidad del nivel freático $(-80 \mathrm{~cm} \pm 20)$ y una baja conductividad hidráulica, como respuesta a la disposición y las características del material acumulado (escarpe), en comparación con los ensayos sin escarpe, sobre turba desnuda, caracterizada por un nivel superficial de agua, proporcionando a las hebras implantadas de Sphagnum una mayor humedad. Un factor importante de considerar en una restauración, es la elección de una capa protectora, con un peso adecuado, que permita disminuir el riesgo de pérdida por evapotranspiración, así como la pérdida de las hebras por los fuertes vientos, característicos de esta región y la introducción de especies exóticas. 


\title{
PLAN DE MANEJO SUSTENTABLE Y MODELO DE FISCALIZACIÓN PARA HUMEDALES CON PREDOMINIO DE MUSGO POMPÓN (SPHAGNUM MAGELLANICUM) EN LAS PROVINCIAS DE LLANQUIHUE Y CHILOÉ
}

\author{
María Francisca Díaz \& Guillermo Delano**
}

La extracción de musgo Sphagnum en humedales de la Región de los Lagos, con fines de exportación, es una actividad que se ha realizado desde 1996. En este período las exportaciones de Sphagnum han aumentado en 29 veces, llegando a 3.900 toneladas de musgo seco y un valor alrededor de 13 millones de dólares FOB/año (2010). La extracción masiva del recurso, asociada a una alta demanda externa, ha contribuido a agotar la disponibilidad del recurso, generando cambios ambientales irreversibles, un impacto negativo sobre la hidrología del lugar y el ciclo de carbono, efectos sobre la biodiversidad, la erosión y el paisaje, calidad de vida de la gente y limitando la posibilidad de proyectar el negocio, tanto para empresas involucradas como para las familias que dependen del recurso. Si bien actualmente no existe un marco regulatorio, ni organismos públicos responsables de regular y controlar la explotación sustentable del recurso, existe gran preocupación e interés de instituciones públicas como ODEPA, SAG, INDAP, CONAF y SEREMI Los Lagos, exportadores, productores y entidades de investigación, por la conservación de éste. En este sentido, en el presente proyecto se propone desarrollar planes de manejo de Sphagnum, con la generación de un producto certificable en cuanto a criterios de sustentabilidad y trazabilidad, con atributos de calidad en función de mercados exigentes de alto valor, y ajustado a buenas prácticas laborales. Para lo anterior, se realizará un diagnóstico participativo con distintos agentes involucrados en la cadena productiva y comercial de musgo Sphagnum. El diagnóstico considera actividades como reuniones técnicas, mesas de trabajo, visitas a terreno, participación de expertos nacionales. Se recolectará información relacionada con sustentabilidad, requerimientos de mercado, condiciones laborales y proceso productivo-comercial. En base a esta información se definirá puntos críticos de intervención a partir de los cuales se desarrollarán propuestas de planes de extracción y manejo sustentable de humedales con predominancia de Sphagnum. En base a la etapa antes indicada, se establecerán ensayos para determinar el crecimiento, desarrollo y regeneración del recurso y aspectos relacionados con manejo y cosecha. Paralelamente se generarán propuestas técnicas que permitan establecer criterios de trazabilidad para evitar contaminación microbiológica y por metales pesados. La propuesta considera generar pautas de manejo compatibles con conceptos de buenas prácticas laborales. A partir de esta información se desarrollarán manuales de manejo y protocolos susceptibles de ser aplicados en procesos de certificación y fiscalización. Los trabajos de investigación en cuanto a crecimiento y desarrollo del musgo se realizarán tanto en condiciones controladas, cámara de crecimiento climatizada (Centro Experimental UST), como en condiciones de campo en predios de agricultores en las provincias de Llanquihue y Chiloé. El resto de los ensayos se realizarán en 4 pomponales ubicados en ambas provincias. Los sitios serán implementados con cercos, secadores, estaciones meteorológicas, entre otros. El programa de transferencias incluye difusión de resultados de los trabajos en sus distintas etapas, (charla informativa al inicio del proyecto, $y$ un seminario de clausura), transferencias de resultados mediante días de campo y cursos a productores, funcionarios públicos y de empresas privadas en temas relacionados con planes de manejo sustentable y protocolos de certificación y fiscalización. Entre los resultados que se espera obtener están: (a) Propuesta de Plan de Manejo que permita la explotación sustentable de humedales con predominio de musgo pompón, (b) Protocolos factibles de ser usados en procesos de certificación y fiscalización de planes de manejo y extracción sustentable, (c) Conocimiento sobre la biología de la especie (Sphagnum magellanicum), que permita desarrollar trabajos relacionados con la preservación de ecosistemas frágiles, como

Universidad Andrés Bello.

Universidad Santo Tomás 
los humedales, (d) Aumento del ingreso y mejoramiento del nivel de vida y condiciones de trabajo de 400 familias de agricultores, e) Generación de competencias a los productores, en cuanto al manejo sustentable de los humedales y de productos con mayor valor agregado, (f) Aplicación de buenas prácticas laborales en faenas de extracción del recurso, (g) Producto de exportación certificable en cuanto a las condiciones de producción sustentable, bajo protocolos de trazabilidad, con mayor valor agregado que el producto actual, que será ofrecido a los mercados de exportación por las Empresas exportadoras involucradas. 


\title{
CARACTERIZACIÓN DE SERVICIOS ECOSISTÉMICOS DE TURBERAS DE LA ISLA GRANDE DE CHILOÉ, UNA HERRAMIENTA PARA LA CONSERVACIÓN
}

\author{
C. León Valdebenito*, G. Oliván Martínez \& E. Fuertes Lasala*
}

Las turberas cumplen un importante rol en el almacenamiento de agua dulce, en la acumulación de carbono y en la conservación de la biodiversidad, entre otros. Sin embargo, el conocimiento sobre estos ecosistemas es muy limitado, especialmente en el sur de Sudamérica. Debido a esto, el presente trabajo tiene como objetivo contribuir al conocimiento a través de la caracterización de dos importantes servicios ecosistémicos: el almacenamiento reciente de carbono y la diversidad briofítica y liquénica en turberas de origen glacial y turberas antropogénicas (o pomponales) presentes en la Isla Grande de Chiloé, con el fin de elaborar una propuesta de pagos por los servicios ecosistémicos prestados, para contribuir a su conservación y desarrollo sostenible. Para cumplir estos objetivos se estudiaron ocho turberas: cinco antropogénicas y 3 de origen glaciar, ubicadas en distintas zonas de la isla. Tras el estudio de la diversidad brio-liquénica se ha determinado un total de 76 especies (35,3 \% endémicas del sur de Sudamérica): 29 musgos, 28 hepáticas y 19 líquenes. Del total, se encontraron 68 especies en las turberas antropogénicas y 38 especies en las de origen glacial. Además, se han reportado 10 nuevos registros para Chiloé y tres nuevos registros para la flora brio-liquénica de Chile. En la estimación de las tasas recientes de acumulación de carbono (RERCA) se registró una tasa media siete veces superior en turberas antropogénicas en relación a las turberas de origen glaciar. Bajo estos resultados, se destaca que la diversidad brio-liquénica presente es alta, con especies poco conocidas y de distribución restringida. Con esto queda patente la importancia que tienen estos ecosistemas en la conservación de la biodiversidad y el significativo aporte que ha supuesto estudiar estos grupos botánicos muy poco conocidos en Chile. Por otra parte, se hace significativo que las tasas de acumulación de carbono en las turberas de Chiloé durante los últimos 100 años han sido considerablemente elevadas al ser comparadas con valores de otras zonas templadas, destacándose especialmente las antropogénicas que presentan las mayores tasas, lo que indica que estos ambientes jóvenes están más activos, tienen mayor capacidad para producir materia orgánica y almacenar carbono. En síntesis, existe evidencia de la significativa cantidad de carbono almacenado en estos sitios, que podría liberarse como $\mathrm{CO}_{2}$, si éstas fueran drenadas. A la luz de nuestros resultados se fundamenta la propuesta de establecer un sistema de pagos por servicios ecosistémicos como forma de compensación de emisiones de $\mathrm{CO}_{2}$ voluntarios, de resguardo de la biodiversidad y reservorio de agua dulce, con los que la población local conseguiría una fuente de ingresos sin necesidad de realizar actividades extractivas, lo que permitiría conservar las turberas, reducir emisiones de $\mathrm{CO}_{2}$ y tener un desarrollo económico sostenible. Dicha propuesta se proyecta como una alternativa viable, ya que distintas autoridades municipales y regionales se han mostrado interesadas en su implementación y además se cuenta con el apoyo de los dueños de algunos predios. En adición a esto, en sondeos iniciales de mercado realizados a empresas chilenas y españolas, éstas han considerado que la propuesta tiene un interesante potencial de desarrollo para acciones de responsabilidad social corporativa. 


\section{NATIONAL INVENTORY OF LANDSCAPES IN SWEDEN (NILS): WETLANDS AND PEATLANDS IN SWEDEN}

Saskia Sandring*

About $20 \%$ of the land area of Sweden is covered by wetlands, of which a large part is peatlands. Even though peatlands are relatively abundant in northern Europe their natural vegetation composition and ecological processes are threatened by hydrological disturbance due to impact from land use and allterrain vehicle tracks, climate change and nutrient deposition. To improve the status of the environment the Swedish government has agreed on 16 environmental quality objectives, each focusing at specific environmental problems or ecosystem types. The Quality objective concerning wetlands focuses on biodiversity, cultural heritage and the recreational value of wetlands. More specifically, the aim is that threatened species have viable populations, that no alien species are introduced, and that no peat harvest occurs at sites of high ecological and nature conservation values. Thus, data are needed in order to measure and evaluate the fulfilment of the environmental quality objectives. One of the intentions of the NILS program is to provide data that can be used to monitor the biodiversity conditions and changes in terrestrial habitats and ecosystems in Sweden, including wetlands and peatlands. The NILS inventory uses a national sample of permanent plots to collect data by remote sensing and field visits. Besides national and international reporting the data are used for governmental strategy planning, policy making and applied research. One example on the application of data obtained by the NILS program is a recent development of an indicator for tree abundance on mires, which is based on data on basal area of trees and their frequency. The phenomenon of increased tree abundance due to climate change, hydrological impact and nutrient deposition has been observed in the whole country. Hence, there is a need to monitor this change and determine cause and effect, as well as to provide the society with representative data for land use and other strategic policy directions. 